\title{
SOME CRITERIA OF BOUNDEDNESS OF $L$-INDEX IN A DIRECTION FOR SLICE HOLOMORPHIC FUNCTIONS IN THE UNIT BALL
}

\author{
Andriy Bandura ${ }^{1}$, Lyubov Shegda ${ }^{2}$, \\ Oleh Skaskiv ${ }^{3}$, Liana Smolovyk ${ }^{4}$ \\ ${ }^{1,2,4}$ Department of Advanced Mathematics \\ Ivano-Frankivsk National Technical University \\ of Oil and Gas \\ 15 Karpatska str., Ivano-Frankivsk - 76019, UKRAINE \\ ${ }^{3}$ Department of Mechanics and Mathematics \\ Ivan Franko National University of Lviv \\ 1 Universytetska str., Lviv - 79000, UKRAINE
}

\begin{abstract}
Let $\mathbf{b} \in \mathbb{C}^{n} \backslash\{\mathbf{0}\}$ be a fixed direction and $\mathbf{L}: \mathbb{B}^{n} \rightarrow \mathbb{R}_{+}$be a positive continuous function such that $L(z)>\frac{\beta|\mathbf{b}|}{1-|z|}$, where $\beta>1$ is some constant. We consider slice holomorphic functions of several complex variables in the unit ball, i.e. we study functions which are analytic in intersection of every slice $\left\{z^{0}+t \mathbf{b}: t \in \mathbb{C}\right\}$ with the unit ball $\mathbb{B}^{n}=\left\{z \in \mathbb{C}^{n}:|z|:=\right.$ $\left.\sqrt{|z|_{1}^{2}+\ldots+\left|z_{n}\right|^{2}}<1\right\}$ for any $z^{0} \in \mathbb{B}^{n}$. For functions from this class we prove some criteria of boundedness of $L$-index in the direction describing local behavior of maximum modulus, minimum modulus of the slice holomorphic function and providing estimates of logarithmic derivative and distribution of zeros. Moreover, we obtain an analog of logarithmic criterion. Note that the hypothesis on holomorphy in one direction together with the hypothesis on joint continuity do not imply holomorphy in whole $n$-dimensional unit ball.
\end{abstract}

AMS Subject Classification: 32A10, 32A17, 32A37, 30H99, 30A05

Key Words: bounded index; bounded $L$-index in direction; slice function; holomorphic function; maximum modulus; minimum modulus; bounded l-index; distribution of zeros; logarithmic derivative; directional derivative; unit ball

Received: February 22, 2021

(C) 2021 Academic Publications

$\S$ Correspondence author 


\section{Introduction}

The paper is an extension of previous work [1]. There it was introduced a concept of $L$-index boundedness in direction for slice holomorphic functions in the unit ball. Also there was deduced some criterion of $L$-index boundedness in direction describing a local behavior of functions from this class. Here we continue these investigations and apply this criterion to deduce more useful criteria of $L$-index boundedness in direction. Among them, there is an logarithmic criterion, an estimate of minimum modulus and an estimate of maximum modulus on a circle obtained by a slice in the unit ball. It should be note that the logarithmic criteria in the case of slice entire functions [2] have applications in analytic theory of differential equations. In general, the concept of bounded index has different applications in value distribution theory [17], summability methods theory [13] and analytic theory of system of differential equations [14].

Let us introduce some notations from [1]. Let $\mathbb{R}_{+}=(0,+\infty), \mathbb{R}_{+}^{*}=[0,+\infty)$, $\mathbf{0}=(0, \ldots, 0), \mathbf{b}=\left(b_{1}, \ldots, b_{n}\right) \in \mathbb{C}^{n} \backslash\{\mathbf{0}\}$ be a given direciton, $\mathbb{B}^{n}=\left\{z \in \mathbb{C}^{n}\right.$ : $|z|<1\}, \mathbb{D}=\{z \in \mathbb{C}:|z|<1\}, L: \mathbb{B}^{n} \rightarrow \mathbb{R}_{+}$be a continuous function such that, for all $z \in \mathbb{B}^{n}$

$$
L(z)>\frac{\beta|\mathbf{b}|}{1-|z|}, \beta=\text { const }>1 .
$$

For a given $z \in \mathbb{B}^{n}$, we denote $S_{z}=\left\{t \in \mathbb{C}: z+t \mathbf{b} \in \mathbb{B}^{n}\right\}$. Clearly, $\mathbb{D}=\mathbb{B}^{1}$.

Let $F: \mathbb{B}^{n} \rightarrow \mathbb{C}$ be an analytic function. The slice functions on $S_{z}$ for a fixed $z^{0} \in \mathbb{B}^{n}$ we will denote as $g_{z^{0}}(t)=F\left(z^{0}+t \mathbf{b}\right)$ and $l_{z^{0}}(t)=L\left(z^{0}+t \mathbf{b}\right)$ for $t \in S_{z}$.

Let $\widetilde{\mathcal{H}}_{\mathbf{b}}\left(\mathbb{B}^{n}\right)$ be the class of functions which are holomorphic on every slices $\left\{z^{0}+t \mathbf{b}: t \in S_{z^{0}}\right\}$ for each $z^{0} \in \mathbb{B}^{n}$ and let $\mathcal{H}_{\mathbf{b}}\left(\mathbb{B}^{n}\right)$ be the class of functions from $\widetilde{\mathcal{H}}_{\mathbf{b}}\left(\mathbb{B}^{n}\right)$ which are joint continuous.

The notation $\partial_{\mathbf{b}} F(z)$ stands for the derivative of the function $g_{z}(t)$ at the point 0 , i.e. for every $p \in \mathbb{N} \quad \partial_{\mathbf{b}}^{p} F(z)=g_{z}^{(p)}(0)$, where $g_{z}(t)=F(z+t \mathbf{b})$ is an analytic function of complex variable $t \in S_{z}$ for a given $z \in \mathbb{C}^{n}$. In this research, we will often call this derivative as directional derivative because if $F$ is an analytic function in $\mathbb{B}^{n}$ then the derivatives of the function $g_{z}(t)$ matches with directional derivatives of the function $F$. Together the hypothesis on joint continuity and the hypothesis on holomorphy in one direction do not imply holomorphy in whole $n$-dimensional unit ball. There were presented some examples to demonstrate it [1] and [3].

A function $F \in \mathcal{H}_{\mathbf{b}}^{n}$ is said [1] to be of bounded L-index in the direction $\mathbf{b}$, 
if there exists $m_{0} \in \mathbb{Z}_{+}$such that for all $m \in \mathbb{Z}_{+}$and each $z \in \mathbb{C}^{n}$ inequality

$$
\frac{\left|\partial_{\mathbf{b}}^{m} F(z)\right|}{m ! L^{m}(z)} \leq \max _{0 \leq k \leq m_{0}} \frac{\left|\partial_{\mathbf{b}}^{k} F(z)\right|}{k ! L^{k}(z)}
$$

is true. The least such integer number $m_{0}$, obeying (2), is called the $L$-index in the direction $\mathbf{b}$ of the function $F$ and is denoted by $N_{\mathbf{b}}\left(F, L, \mathbb{B}^{n}\right)$. If such $m_{0}$ does not exist then we put $N_{\mathbf{b}}(F, L)=\infty$, and the function $F$ is called of unbounded $L$-index in the direction $\mathbf{b}$ in this case. If $L(z) \equiv 1$ then the function $F$ is said to be of bounded index in the direction $\mathbf{b}$ and $N_{\mathbf{b}}(F)=N_{\mathbf{b}}(F, 1)$ is called the index in the direction $\mathbf{b}$. Let $l: \mathbb{D} \rightarrow \mathbb{R}_{+}$be a continuous function such that $l(z)>\frac{\beta}{1-z}$. For $n=1, \mathbf{b}=1, L(z) \equiv l(z)(z \in \mathbb{D})$ the inequality (2) defines an analytic function in the unit disc of bounded $l$-index with the $l$-index $N(F, l) \equiv N_{1}(F, l)$ (see [16]). Let $N_{\mathbf{b}}\left(F, L, z^{0}\right)$ stands for the $L$-index in the direction $\mathbf{b}$ of the function $F$ at the point $z^{0}$, i.e., it is the least integer $m_{0}$, for which inequality (2) is satisfied at the point $z=z^{0}$. By analogy, the notation $N\left(f, l, z^{0}\right)$ is defined if $n=1$, i.e. in the case of functions of one variable.

Note that the positivity and continuity of the function $L$ are weak restrictions to deduce constructive results. Thus, we assume additional restrictions by the function $L$.

Let us denote

$$
\begin{gathered}
\lambda_{\mathbf{b}}(\eta)= \\
=\sup _{z \in \mathbb{B}^{n}} \sup _{t_{1}, t_{2} \in S_{z}}\left\{\frac{L\left(z+t_{1} \mathbf{b}\right)}{L\left(z+t_{2} \mathbf{b}\right)}:\left|t_{1}-t_{2}\right| \leq \frac{\eta}{\min \left\{L\left(z+t_{1} \mathbf{b}\right), L\left(z+t_{2} \mathbf{b}\right)\right\}}\right\} .
\end{gathered}
$$

By $Q_{\mathbf{b}}\left(\mathbb{B}^{n}\right)$, we denote a class of positive continuous functions $L: \mathbb{B}^{n} \rightarrow \mathbb{R}_{+}$, satisfying the condition

$$
\forall \eta \in[0 ; \beta]: \lambda_{\mathbf{b}}(\eta)<+\infty .
$$

For a positive continuous function $l(t), t \in \mathbb{D}$, and $\eta>0$ we define $\lambda(\eta) \equiv$ $\lambda_{1}^{\mathbf{b}}(\eta)$ in the cases when $\mathbf{b}=1, n=1, L \equiv l$. Let $Q \equiv Q_{1}^{1}$ be a class of positive continuous functions $l(t), t \in \mathbb{D}$, obeying the condition $0<\lambda(\eta)<+\infty$ for all $\eta \in[0 ; \beta]$.

We need the following statements from [1].

Proposition 1 ([1]). Let $L \in Q_{\mathbf{b}}\left(\mathbb{B}^{n}\right), \frac{1}{\beta}<\theta_{1} \leq \theta_{2}<+\infty, \theta_{1} L(z) \leq$ $L^{*}(z) \leq \theta_{2} L(z)$. A function $F \in \widetilde{\mathcal{H}}_{\mathbf{b}}\left(\mathbb{B}^{n}\right)$ is of bounded $L^{*}$-index in the direction $\mathbf{b}$ if and only if $F$ is of bounded $L$-index in the direction $\mathbf{b}$. 
Proposition 2. Let $L \in Q_{\mathbf{b}}\left(\mathbb{B}^{n}\right), m \in \mathbb{C} \backslash\{0\}$. A function $F \in \widetilde{\mathcal{H}}_{\mathbf{b}}\left(\mathbb{B}^{n}\right)$ is of a bounded $L$-index in the direction $\mathbf{b} \in \mathbb{C}^{n}$ if and only if $F(z)$ is of a bounded $L$-index in the direction $m \mathbf{b}$.

Theorem 3 ([1]). Let $L \in Q_{\mathbf{b}}\left(\mathbb{B}^{n}\right)$. A function $F \in \widetilde{\mathcal{H}}_{\mathbf{b}}\left(\mathbb{B}^{n}\right)$ is of bounded $L$-index in the direction $\mathbf{b}$ if and only if for each $\eta \in(0 ; \beta]$, there exist $n_{0}=$ $n_{0}(\eta) \in \mathbb{Z}_{+}$and $P_{1}=P_{1}(\eta) \geq 1$ such that, for every $z \in \mathbb{B}^{n}$, there exists $k_{0}=k_{0}(z) \in \mathbb{Z}_{+}, 0 \leq k_{0} \leq n_{0}$, and

$$
\max \left\{\left|\partial_{\mathbf{b}}^{k_{0}} F(z+t \mathbf{b})\right|:|t| \leq \frac{\eta}{L(z)}\right\} \leq P_{1}\left|\partial_{\mathbf{b}}^{k_{0}} F(z)\right| .
$$

\section{Estimate of maximum modulus by minimum modulus}

Using Theorem 3, we will prove the next criterion of $L$-index boundedness in direction. Similar results was firstly deduced by Fricke [11] for entire functions bounded index. Further it was generalized for various classes of holomorphic functions $[15,6]$.

Theorem 4. Let $L \in Q_{\mathbf{b}}\left(\mathbb{B}^{n}\right)$. A function $F \in \widetilde{\mathcal{H}}_{\mathbf{b}}\left(\mathbb{B}^{n}\right)$ has bounded $L$-index in a direction $\mathbf{b} \in \mathbb{C}^{n} \backslash\{\mathbf{0}\}$ if and only if for any $r_{1}$ and any $r_{2}$ with $0<r_{1}<r_{2} \leq \beta$, there exists number $P_{1}=P_{1}\left(r_{1}, r_{2}\right) \geq 1$ such that for each $z^{0} \in \mathbb{B}^{n}$

$$
\begin{gathered}
\max \left\{\left|F\left(z^{0}+t \mathbf{b}\right)\right|:|t|=r_{2} / L\left(z^{0}\right)\right\} \\
\leq P_{1} \max \left\{\left|F\left(z^{0}+t \mathbf{b}\right)\right|:|t|=r_{1} / L\left(z_{0}\right)\right\} .
\end{gathered}
$$

Proof. Our proof is based on the proof of appropriate theorem for slice entire functions of bounded $L$-index in direction [4] and for analytic functions in the unit ball [7].

Necessity. Let $N_{\mathbf{b}}\left(F, L, \mathbb{B}^{n}\right)<+\infty$. On the contrary, we assume that there exist numbers $r_{1}$ and $r_{2}, 0<r_{1}<r_{2} \leq \beta$, such that for every $P_{*} \geq 1$ there exist $z^{*}=z^{*}\left(P_{*}\right) \in \mathbb{B}^{n}$, for which the following inequality is valid

$$
\begin{gathered}
\max \left\{\left|F\left(z^{*}+t \mathbf{b}\right)\right|:|t|=r_{2} / L\left(z^{*}\right)\right\} \\
>P_{*} \max \left\{\left|F\left(z^{*}+t \mathbf{b}\right)\right|:|t|=r_{1} / L\left(z^{*}\right)\right\} .
\end{gathered}
$$


By Theorem 3 there exist $n_{0}=n_{0}\left(r_{2}\right) \in \mathbb{Z}_{+}$and $P_{0}=P_{0}\left(r_{2}\right) \geq 1$ such that for every $z^{*} \in \mathbb{B}^{n}$ and some $k_{0}=k_{0}\left(z^{*}\right) \in \mathbb{Z}_{+}, 0 \leq k_{0} \leq n_{0}$, one has

$$
\max \left\{\left|\partial_{\mathbf{b}}^{k_{0}} F\left(z^{*}+t \mathbf{b}\right)\right|:|t|=r_{2} / L\left(z^{*}\right)\right\} \leq P_{0}\left|\partial_{\mathbf{b}}^{k_{0}} F\left(z^{*}\right)\right| .
$$

We remark that for $k_{0}=0$ the proof of necessity is obvious because (6) yields $\max \left\{\left|F\left(z^{*}+t \mathbf{b}\right)\right|:|t|=r_{2} / L\left(z^{*}\right)\right\} \leq P_{0}\left|F\left(z^{*}\right)\right| \leq P_{0} \max \left\{\left|F\left(z^{*}+t \mathbf{b}\right)\right|:|t|=\right.$ $\left.r_{1} / L\left(z^{*}\right)\right\}$.

Suppose that $k_{0}>0$. Put

$$
P_{*}=n_{0} !\left(\frac{r_{2}}{r_{1}}\right)^{n_{0}}\left(P_{0}+\frac{r_{1}}{r_{2}-r_{1}}\right)+1 .
$$

Let $t_{0} \in S_{z^{*}}$ be such that $\left|t_{0}\right|=\frac{r_{1}}{L\left(z^{*}\right)}$ and

$$
\left|F\left(z^{*}+t_{0} \mathbf{b}\right)\right|=\max \left\{\left|F\left(z^{*}+t \mathbf{b}\right)\right|:|t|=\frac{r_{1}}{L\left(z^{*}\right)}\right\}>0,
$$

and $t_{0 j} \in S_{z^{*}},\left|t_{0 j}\right|=r_{2} / L\left(z^{*}\right)$, be such that $\left|\partial_{\mathbf{b}}^{j} F\left(z^{*}+t_{0 j} \mathbf{b}\right)\right|=\max \left\{\left|\partial_{\mathbf{b}}^{j} F\left(z^{*}+t \mathbf{b}\right)\right|\right.$ : $\left.|t|=r_{2} / L\left(z^{*}\right)\right\}, j \in \mathbb{Z}_{+}$. In the case $\left|F\left(z^{*}+t_{0} \mathbf{b}\right)\right|=0$ by the uniqueness theorem for all $t \in S_{z^{*}}$ we obtain $F\left(z^{*}+t \mathbf{b}\right) \equiv 0$. However, it contradicts inequality (5). By Cauchy's inequality we have

$$
\begin{array}{r}
\frac{\left|\partial_{\mathbf{b}}^{j} F\left(z^{*}\right)\right|}{j !} \leq\left(\frac{L\left(z^{*}\right)}{r_{1}}\right)^{j}\left|F\left(z^{*}+t_{0} \mathbf{b}\right)\right|, j \in \mathbb{Z}_{+}, \\
\left|\partial_{\mathbf{b}}^{j} F\left(z^{*}+t_{0 j} \mathbf{b}\right)-\partial_{\mathbf{b}}^{j} F\left(z^{*}\right)\right|=\left|\int_{0}^{t_{0 j}} \partial_{\mathbf{b}}^{j+1} F\left(z^{*}+t \mathbf{b}\right) d t\right| \\
\leq\left|\partial_{\mathbf{b}}^{j+1} F\left(z^{*}+t_{0(j+1)} \mathbf{b}\right)\right| \frac{r_{2}}{L\left(z^{*}\right)} .
\end{array}
$$

From (8) and (9) we have

$$
\begin{aligned}
& \left|\partial_{\mathbf{b}}^{j+1} F\left(z^{*}+t_{0(j+1)} \mathbf{b}\right)\right| \geq \frac{L\left(z^{*}\right)}{r_{2}}\left\{\left|\partial_{\mathbf{b}}^{j} F\left(z^{*}+t_{0 j} \mathbf{b}\right)\right|-\left|\partial_{\mathbf{b}}^{j} F\left(z^{*}\right)\right|\right\} \\
& \geq \frac{L\left(z^{*}+t^{*} \mathbf{b}\right)}{r_{2}}\left|\partial_{\mathbf{b}}^{j} F\left(z^{*}+t_{0 j} \mathbf{b}\right)\right|-\frac{j ! L^{j+1}\left(z^{*}\right)}{r_{2}\left(r_{1}\right)^{j}}\left|F\left(z^{*}+t_{0} \mathbf{b}\right)\right|,
\end{aligned}
$$

where $j \in \mathbb{Z}_{+}$. Hence, for $k_{0} \geq 1$ we get

$$
\left|\partial_{\mathbf{b}}^{k_{0}} F\left(z^{*}+t_{0 k_{0}} \mathbf{b}\right)\right| \geq \frac{L\left(z^{*}\right)}{r_{2}}\left|\partial_{\mathbf{b}}^{k_{0}-1} F\left(z^{*}+t_{0\left(k_{0}-1\right)} \mathbf{b}\right)\right|
$$




$$
\begin{gathered}
-\frac{\left(k_{0}-1\right) ! L^{k_{0}}\left(z^{*}\right)}{r_{2}\left(r_{1}\right)^{k_{0}-1}}\left|F\left(z^{*}+t_{0} \mathbf{b}\right)\right| \geq \ldots \geq \frac{L^{k_{0}}\left(z^{*}\right)}{\left(r_{2}\right)^{k_{0}}}\left|F\left(z^{*}+t_{00} \mathbf{b}\right)\right| \\
-\left(\frac{0 !}{\left(r_{2}\right)^{k_{0}}}+\frac{1 !}{\left(r_{2}\right)^{k_{0}-1} r_{1}}+\ldots+\frac{\left(k_{0}-1\right) !}{r_{2}\left(r_{1}\right)^{k_{0}-1}}\right) L^{k_{0}}\left(z^{*}\right)\left|F\left(z^{*}+t_{0} \mathbf{b}\right)\right| \\
=\frac{L^{k_{0}}\left(z^{*}\right)}{\left(r_{2}\right)^{k_{0}}}\left|F\left(z^{*}+t_{0} \mathbf{b}\right)\right|\left(\frac{\left|F\left(z^{*}+t_{00} \mathbf{b}\right)\right|}{\left|F\left(z^{*}+t_{0} \mathbf{b}\right)\right|}-\sum_{j=0}^{k_{0}-1} j !\left(\frac{r_{2}}{r_{1}}\right)^{j}\right) .
\end{gathered}
$$

In view of (5) we have $\left|F\left(z^{*}+t_{00} \mathbf{b}\right)\right| /\left|F\left(z^{*}+t_{0} \mathbf{b}\right)\right|>P_{*}$. Besides, this inequality holds

$$
\sum_{j=0}^{k_{0}-1} j !\left(\frac{r_{2}}{r_{1}}\right)^{j} \leq k_{0} !\left(\frac{\left(r_{2} / r_{1}\right)^{k_{0}}-1}{r_{2} / r_{1}-1}\right) \leq n_{0} ! \frac{r_{1}}{r_{2}-r_{1}}\left(\frac{r_{2}}{r_{1}}\right)^{n_{0}} .
$$

Applying (7), we obtain

$$
\frac{\left|F\left(z^{*}+t_{00} \mathbf{b}\right)\right|}{\left|F\left(z^{*}+t_{0} \mathbf{b}\right)\right|}-\sum_{j=0}^{k_{0}-1} j ! \frac{r_{2}^{j}}{r_{1}^{j}}>P_{*}-\frac{n_{0} ! r_{1}}{r_{2}-r_{1}}\left(\frac{r_{2}}{r_{1}}\right)^{n_{0}}=n_{0} !\left(\frac{r_{2}}{r_{1}}\right)^{n_{0}} P_{0}+1 .
$$

It follows from (10), (6) and (8) that

$$
\begin{aligned}
& \left|\partial_{\mathbf{b}}^{k_{0}} F\left(z^{*}+t_{0 k_{0}} \mathbf{b}\right)\right|>\frac{L^{k_{0}}\left(z^{*}\right)}{\left(r_{2}\right)^{k_{0}}}\left(P_{*}-n_{0} ! \frac{r_{1}}{r_{2}-r_{1}}\left(\frac{r_{2}}{r_{1}}\right)^{n_{0}}\right)\left(\frac{r_{1}}{L\left(z^{*}\right)}\right)^{k_{0}} \\
& \times \frac{\left|\partial_{\mathbf{b}}^{k_{0}} F\left(z^{*}\right)\right|}{k_{0} !} \geq\left(\frac{r_{1}}{r_{2}}\right)^{n_{0}}\left(P_{*}-n_{0} ! \frac{r_{1}}{r_{2}-r_{1}}\left(\frac{r_{2}}{r_{1}}\right)^{n_{0}}\right) \frac{\left|\partial_{\mathbf{b}}^{k_{0}} F\left(z^{*}+t_{0 k_{0}} \mathbf{b}\right)\right|}{n_{0} ! P_{0}} .
\end{aligned}
$$

Hence, $P_{*}<n_{0} !\left(\frac{r_{2}}{r_{1}}\right)^{n_{0}}\left(P_{0}+\frac{r_{1}}{r_{2}-r_{1}}\right)$ which contradicts $(7)$.

Sufficiency. We choose any two numbers $r_{1} \in(0,1)$ and $r_{2} \in(1, \beta)$. For a given $z^{0} \in \mathbb{B}^{n}$ we expand the function $g_{z^{0}}(t)=F\left(z^{0}+t \mathbf{b}\right)$ in a power series in the disc $\left\{t:|t| \leq \beta / L\left(z^{0}\right)\right\} \subset S_{z^{0}} F\left(z^{0}+t \mathbf{b}\right)=\sum_{m=0}^{\infty} b_{m}\left(z^{0}\right) t^{m}$, where $b_{m}\left(z^{0}\right)=$ $\partial_{\mathbf{b}}^{m} F\left(z^{0}\right) / m$ !. For $r \leq \frac{\beta}{L\left(z^{0}\right)}$ we denote

$$
\begin{gathered}
M_{\mathbf{b}}\left(r, z^{0}, F\right)=\max \left\{\left|F\left(z^{0}+t \mathbf{b}\right)\right|:|t|=r\right\}, \\
\mu_{\mathbf{b}}\left(r, z^{0}, F\right)=\max \left\{\left|b_{m}\left(z^{0}\right)\right| r^{m}: m \geq 0\right\}, \\
\nu_{\mathbf{b}}\left(r, z^{0}, F\right)=\max \left\{\left|b_{m}\left(z^{0}\right)\right| r^{m}:\left|b_{m}\left(z^{0}\right)\right| r^{m}=\mu_{\mathbf{b}}\left(r, z^{0}, F\right)\right\} .
\end{gathered}
$$


By Cauchy's inequality $\mu_{\mathbf{b}}\left(r, z^{0}, F\right) \leq M_{\mathbf{b}}\left(r, z^{0}, F\right)$. But for $r=1 / L\left(z^{0}\right)$ we have

$$
M_{\mathbf{b}}\left(r_{1} r, z^{0}, F\right) \leq \sum_{m=0}^{\infty}\left|b_{m}\left(z^{0}\right)\right| r^{m} r_{1}^{m} \leq \mu_{\mathbf{b}}\left(r, z^{0}, F\right) \sum_{m=0}^{\infty} r_{1}^{m}=\frac{\mu_{\mathbf{b}}\left(r, z^{0}, F\right)}{1-r_{1}} .
$$

Since $\nu_{\mathbf{b}}\left(r, z^{0}, F\right)$ is monotone in $r$, we deduce

$$
\begin{aligned}
\ln \mu_{\mathbf{b}}\left(r_{2} r, z^{0}, F\right)- & \ln \mu_{\mathbf{b}}\left(r, z^{0}, F\right)=\int_{r}^{r_{2} r} \frac{\nu_{\mathbf{b}}\left(t, z^{0}, F\right)}{t} d t \\
& \geq \nu_{\mathbf{b}}\left(r, z^{0}, F\right) \ln r_{2} .
\end{aligned}
$$

Hence,

$$
\begin{gathered}
\nu_{\mathbf{b}}\left(r, z^{0}, F\right) \leq \frac{1}{\ln r_{2}}\left(\ln \mu_{\mathbf{b}}\left(r_{2} r, z^{0}, F\right)-\ln \mu_{\mathbf{b}}\left(r, z^{0}, F\right)\right) \\
\leq \frac{1}{\ln r_{2}}\left\{\ln M_{\mathbf{b}}\left(r_{2} r, z^{0}, F\right)-\ln \left(\left(1-r_{1}\right) M_{\mathbf{b}}\left(r_{1} r, z^{0}, F\right)\right)\right\} \\
\left.=-\frac{\ln \left(1-r_{1}\right)}{\ln r_{2}}+\frac{1}{\ln r_{2}}\left\{\ln M_{\mathbf{b}}\left(r_{2} r, z^{0}, F\right)-\ln M_{\mathbf{b}}\left(r_{1} r, z^{0}, F\right)\right)\right\} .
\end{gathered}
$$

Let $N_{\mathbf{b}}\left(F, L, z^{0}\right)$ be the $L$-index in the direction $\mathbf{b}$ of the function $F$ at the point $z^{0}$. It is obvious that

$$
N_{\mathbf{b}}\left(F, L, z^{0}\right) \leq \nu_{\mathbf{b}}\left(1 / L\left(z^{0}\right), z^{0}, F\right)=\nu_{\mathbf{b}}\left(r, z^{0}, F\right) .
$$

However, inequality (4) can be written in the following form

$$
M_{\mathbf{b}}\left(\frac{r_{2}}{L\left(z^{0}\right)}, z^{0}, F\right) \leq P_{1}\left(r_{1}, r_{2}\right) M_{\mathbf{b}}\left(\frac{r_{1}}{L\left(z^{0}\right)}, z^{0}, F\right) .
$$

Thus, from (11) we obtain $N_{\mathbf{b}}\left(z^{0}, L, F\right) \leq-\frac{\ln \left(1-r_{1}\right)}{\ln r_{2}}+\frac{\ln P_{1}\left(r_{1}, r_{2}\right)}{\ln r_{2}}$ for every $z^{0} \in$ $\mathbb{C}^{n}$, i.e. $N_{\mathbf{b}}(F, L) \leq-\frac{\ln \left(1-r_{1}\right)}{\ln r_{2}}+\frac{\ln P_{1}\left(r_{1}, r_{2}\right)}{\ln r_{2}}$. Theorem 4 is proved.

In view of proof of sufficiency in Theorem 4 the following lemma is valid.

Lemma 5. Let $L \in Q_{\mathbf{b}}\left(\mathbb{B}^{n}\right), F \in \widetilde{\mathcal{H}}_{\mathbf{b}}\left(\mathbb{B}^{n}\right)$. If there exist numbers $r_{1}$ and $r_{2}, 0<r_{1}<1<r_{2} \leq \beta$, and $P_{1} \geq 1$ such that for every $z^{0} \in \mathbb{B}^{n}$ inequality (4) holds then the function $F$ is of bounded $L$-index in the direction $\mathbf{b}$.

We can relax sufficient conditions of Lemma 5, replacing the condition $0<$ $r_{1}<1<r_{2} \leq \beta$ by $0<r_{1}<r_{2}<+\infty$. 
Theorem 6. Let $L \in Q_{\mathbf{b}}\left(\mathbb{B}^{n}\right)$ and $F \in \widetilde{\mathcal{H}}_{\mathbf{b}}\left(\mathbb{B}^{n}\right)$. If there exist $r_{1}$ and $r_{2}$, $0<r_{1}<r_{2} \leq \beta$, and $P_{1} \geq 1$ such that for all $z^{0} \in \mathbb{B}^{n}$ inequality (4) is satisfied then the function $F$ is of bounded $L$-index in the direction $\mathbf{b}$.

Proof. Our proof is based on idea of Kuzyk and Sheremeta [12]. They proposed this method to investigate the $l$-index boundedness of entire solutions of linear differential equations. Later their idea was applied for entire functions of bounded $L$-index in the direction [8].

Inequality (4) for $0<r_{1}<r_{2}<\beta$ implies

$$
\begin{gathered}
\max \left\{\left|F\left(z^{0}+t \mathbf{b}\right)\right|:|t|=\frac{2 r_{2}}{r_{1}+r_{2}} \frac{r_{1}+r_{2}}{2 L\left(z^{0}\right)}\right\} \\
\leq P_{1} \max \left\{\left|F\left(z^{0}+t \mathbf{b}\right)\right|:|t|=\frac{2 r_{1}}{r_{1}+r_{2}} \frac{r_{1}+r_{2}}{2 L\left(z_{0}\right)}\right\} .
\end{gathered}
$$

Putting $L^{*}(z)=\frac{2 L(z)}{r_{1}+r_{2}}$, we obtain

$$
\begin{gathered}
\max \left\{\left|F\left(z^{0}+t \mathbf{b}\right)\right|:|t|=\frac{2 r_{2}}{\left(r_{1}+r_{2}\right) L^{*}\left(z^{0}\right)}\right\} \\
\leq P_{1} \max \left\{\left|F\left(z^{0}+t \mathbf{b}\right)\right|:|t|=\frac{2 r_{1}}{\left(r_{1}+r_{2}\right) L^{*}\left(z^{0}\right)}\right\},
\end{gathered}
$$

where $0<\frac{2 r_{1}}{r_{1}+r_{2}}<1<\frac{2 r_{2}}{r_{1}+r_{2}}<\frac{2 \beta}{r_{1}+r_{2}}$. Clearly, $L^{*}(z)=\frac{2 L(z)}{r_{1}+r_{2}}>\frac{2 \beta|b|}{\left(r_{1}+r_{2}\right)(1-|z|)}$, i.e., $L^{*}$ satisfies $(1)$ and belongs to the class $Q_{\mathbf{b}}\left(\mathbb{B}^{n}\right)$ with $\frac{2 \beta}{r_{1}+r_{2}}$ instead of the $\beta$. From the validity of inequality (12) we get that by Lemma 5 the function $F$ has bounded $L^{*}$-index in the direction $\mathbf{b}$. And by Proposition 1 the function $F$ has bounded $L$-index in the direction $\mathbf{b}$.

The following theorem gives an estimate of maximum modulus by minimum modulus. It was firstly obtained by Fricke [11] for entire functions of bounded index.

Theorem 7. Let $L \in Q_{\mathbf{b}}\left(\mathbb{B}^{n}\right)$. If a function $F \in \widetilde{\mathcal{H}}_{\mathbf{b}}\left(\mathbb{B}^{n}\right)$ is of bounded $L$-index in the direction $\mathbf{b}$ then for each $R, 0<R \leq \beta$, there exist $P_{2}(R) \geq 1$ and $\eta(R) \in(0, R)$ such that for every $z^{0} \in \mathbb{C}^{n}$ and some $r=r\left(z^{0}\right) \in[\eta(R), R]$ the inequality holds

$$
\max \left\{\left|F\left(z^{0}+t \mathbf{b}\right)\right|:|t|=r / L\left(z^{0}\right)\right\} \leq P_{2} \min \left\{\left|F\left(z^{0}+t \mathbf{b}\right)\right|:|t|=r / L\left(z^{0}\right)\right\} .
$$


Proof. Our proof is based on the proof of appropriate theorem for analytic functions in the unit ball [7].

Let $N_{\mathbf{b}}(F, L)=N<+\infty$ and $R \geq 0$. We put

$$
R_{0}=1, r_{0}=\frac{R}{8(R+1)}, R_{j}=\frac{R_{j-1}}{4 N} r_{j-1}^{N}, r_{j}=\frac{1}{8} R_{j}(j=1,2, \ldots, N) .
$$

Let $z^{0} \in \mathbb{B}^{n}$, and $N_{0}=N_{\mathbf{b}}\left(z^{0}, L, F\right)$ be the $L$-index in the direction $\mathbf{b}$ of the function $F$ at the point $z^{0}$, i.e. $N_{\mathbf{b}}\left(z^{0}, L, F\right)$ be the least number $m_{0}$, for which inequality (2) holds with $z=z^{0}$. The maximum in the right part of (2) is attained at $m_{0}$. Obviously, $0 \leq N_{0} \leq N$. For $z^{0} \in \mathbb{B}^{n}$ we develop $F\left(z^{0}+t \mathbf{b}\right)$ in series by powers $t$

$$
F\left(z^{0}+t \mathbf{b}\right)=\sum_{m=0}^{\infty} b_{m}\left(z^{0}\right) t^{m}, b_{m}\left(z^{0}\right)=\frac{\partial_{\mathbf{b}}^{m} F\left(z^{0}\right)}{m !} .
$$

We put $a_{m}\left(z^{0}\right)=\frac{\left|b_{m}\left(z^{0}\right)\right|}{L^{m}\left(z^{0}\right)}=\frac{\left|\partial_{\mathbf{b}}^{m} F\left(z^{0}\right)\right|}{m ! L^{m}\left(z^{0}\right)}$. For any $m \in \mathbb{Z}_{+}$the inequality $a_{N_{0}}\left(z^{0}\right) \geq$ $a_{m}\left(z^{0}\right)=R_{0} a_{m}\left(z^{0}\right)$ holds. There exists the least number $n_{0} \in\left\{0,1, \ldots, N_{0}\right\}$ such that for all $m \in \mathbb{Z}_{+} a_{n_{0}}\left(z^{0}\right) \geq a_{m}\left(z^{0}\right) R_{N_{0}-n_{0}}$.

Thus, $a_{n_{0}}\left(z^{0}\right) \geq a_{N_{0}}\left(z^{0}\right) R_{N_{0}-n_{0}}$ and $a_{j}\left(z^{0}\right)<a_{N_{0}}\left(z^{0}\right) R_{N_{0}-j}$ for $j<n_{0}$, because if $a_{j_{0}}\left(z^{0}\right) \geq a_{N_{0}}\left(z^{0}\right) R_{N_{0}-j_{0}}$ for some $j_{0}<n_{0}$, then $a_{j_{0}}\left(z^{0}\right) \geq a_{m}\left(z^{0}\right) R_{N_{0}-j_{0}}$ for all $m \in \mathbb{Z}_{+}$and it contradicts the choice of $n_{0}$. In view of $a_{j}\left(z^{0}\right)<$ $a_{N_{0}}\left(z^{0}\right) R_{N_{0}-j}\left(j<n_{0}\right)$ and $a_{m}\left(z^{0}\right) \leq a_{N_{0}}\left(z^{0}\right)\left(m>n_{0}\right)$ for $t \in S_{z^{0}}$ and $|t|=\frac{1}{L\left(z^{0}\right)} r_{N_{0}-n_{0}}$ we have

$$
\begin{gathered}
\left|F\left(z^{0}+t \mathbf{b}\right)\right|=\left|b_{n_{0}}\left(z^{0}\right) t^{n_{0}}+\sum_{m \neq n_{0}} b_{m}\left(z^{0}\right) t^{m}\right| \\
\geq\left|b_{n_{0}}\left(z^{0}\right)\right||t|^{n_{0}}-\sum_{m \neq n_{0}}\left|b_{m}\left(z^{0}\right)\right||t|^{m} \\
=a_{n_{0}}\left(z^{0}\right) r_{N_{0}-n_{0}}^{n_{0}}-\sum_{m \neq 0} a_{m}\left(z^{0}\right) r_{N_{0}-n_{0}}^{m} \\
=a_{n_{0}}\left(z^{0}\right) r_{N_{0}-n_{0}}^{n_{0}}-\sum_{j<n_{0}} a_{j}\left(z^{0}\right) r_{N_{0}-n_{0}}^{j}-\sum_{m>n_{0}} a_{m}\left(z^{0}\right) r_{N_{0}-n_{0}}^{m} \\
\geq a_{N_{0}}\left(z^{0}\right) R_{N_{0}-n_{0}} r_{N_{0}-n_{0}}^{n_{0}} \sum_{m>n_{0}} a_{N_{0}}\left(z^{0}\right) r_{N_{0}-n_{0}}^{m} \\
\geq a_{N_{0}}\left(z^{0}\right) R_{N_{0}-n_{0}} r_{N_{0}-n_{0}}^{n_{0}}-n_{0} a_{N_{0}}\left(z^{0}\right) R_{N_{0}-n_{0}+1}-\frac{a_{N_{0}}\left(z^{0}\right) r_{N_{0}-n_{0}}^{n_{0}+1}}{1-r_{N_{0}-n_{0}}}
\end{gathered}
$$




$$
\begin{gathered}
=a_{N_{0}}\left(z^{0}\right)\left(R_{N_{0}-n_{0}} r_{N_{0}-n_{0}}^{n_{0}}-\frac{n_{0}}{4 N} R_{N_{0}-n_{0}} r_{N_{0}-n_{0}}^{N}-r_{N_{0}-n_{0}}^{n_{0}} \frac{r_{N_{0}-n_{0}}}{1-r_{N_{0}-n_{0}}}\right) \\
\geq a_{N_{0}}\left(z^{0}\right)\left(R_{N_{0}-n_{0}} r_{N_{0}-n_{0}}^{n_{0}}-\frac{1}{4} R_{N_{0}-n_{0}} r_{N_{0}-n_{0}}^{n_{0}}-\frac{1}{4} R_{N_{0}-n_{0}} r_{N_{0}-n_{0}}^{n_{0}}\right) \\
=\frac{1}{2} a_{N_{0}}\left(z^{0}\right) R_{N_{0}-n_{0}} r_{N_{0}-n_{0}}^{n_{0}} .
\end{gathered}
$$

For $t \in S_{z^{0}}$ we also have

$$
\begin{gathered}
\left|F\left(z^{0}+t \mathbf{b}\right)\right| \leq \sum_{m=0}^{+\infty}\left|b_{m}\left(z^{0}\right)\right||t|^{m}=\sum_{m=0}^{\infty} a_{m}\left(z^{0}\right) r_{N_{0}-n_{0}}^{m} \\
\leq a_{N_{0}}\left(z^{0}\right) \sum_{m=0}^{+\infty} r_{N_{0}-n_{0}}^{m}=\frac{a_{N_{0}}\left(z^{0}\right)}{1-r_{N_{0}-n_{0}}} \leq \frac{a_{N_{0}}\left(z^{0}\right)}{1-1 / 8}=\frac{8}{7} a_{N_{0}}\left(z^{0}\right) .
\end{gathered}
$$

From (14) and (15) we obtain

$$
\begin{aligned}
& \max \left\{\left|F\left(z^{0}+t \mathbf{b}\right)\right|:|t|=r_{N_{0}-n_{0}} / L\left(z^{0}\right)\right\} \leq \frac{8}{7} a_{N_{0}}\left(z^{0}\right) \\
& \leq \frac{16}{7} \frac{1}{R_{N_{0}-n_{0}}} r_{N_{0}-n_{0}}^{-n_{0}} \min \left\{\left|F\left(z^{0}+t \mathbf{b}\right)\right|:|t|=\frac{r_{N_{0}-n_{0}}}{L\left(z^{0}\right)}\right\} \\
& \leq \frac{16}{7} \frac{1}{R_{N}} r_{N}^{-N} \min \left\{\left|F\left(z^{0}+t \mathbf{b}\right)\right|:|t|=r_{N_{0}-n_{0}} / L\left(z^{0}\right)\right\},
\end{aligned}
$$

i.e. (13) holds with $P_{2}(R)=\frac{16}{7 R_{N} r_{N}^{N}}, \eta(R)=r_{N}=\frac{1}{8 R_{N}}$ and $r=r_{N_{0}-n_{0}}$.

Below we will prove the sufficient conditions which are partially symmetric to necessary conditions from Theorem 7 .

Theorem 8. Let $L \in Q_{\mathbf{b}}\left(\mathbb{B}^{n}\right), F \in \widetilde{\mathcal{H}}_{\mathbf{b}}\left(\mathbb{B}^{n}\right)$. If there exists $R \in(0, \beta / 2)$ (or if there exists $R \in[\beta / 2, \beta)$ and $\left(\forall z \in \mathbb{B}^{n}\right): L(z)>\frac{2 \beta|b|}{1-|z|}$ ) and there exist $P_{2} \geq 1, \eta \in(0, R)$ such that for all $z^{0} \in \mathbb{B}^{n}$ and some $r=r\left(z^{0}\right) \in[\eta, R]$ inequality (13) holds, then the function $F$ has bounded $L$-index in the direction b.

Proof. Our proof is based on the proof of appropriate proposition for analytic functions of bounded $L$-index in direction [5]. In view of Theorem 6 we need to show existence $P_{1}$ such that for all $z^{0} \in \mathbb{B}^{n}$

$$
\max \left\{\left|F\left(z^{0}+t \mathbf{b}\right)\right|:|t|=(\beta-R) / L\left(z^{0}\right)\right\}
$$




$$
\leq P_{1} \max \left\{\left|F\left(z^{0}+t \mathbf{b}\right)\right|:|t|=R / L\left(z^{0}\right)\right\} .
$$

Assume that there exist $R \in(0, \beta / 2), P_{2} \geq 1$ and $\eta \in(0, R)$ such that for every $z^{0} \in \mathbb{B}^{n}$ and some $r=r\left(z^{0}\right) \in[\eta, R]$ we have

$$
\begin{gathered}
\max \left\{\left|F\left(z^{0}+t \mathbf{b}\right)\right|:|t|=r / L\left(z^{0}\right)\right\} \\
\leq P_{2} \min \left\{\left|F\left(z^{0}+t \mathbf{b}\right)\right|:|t|=r / L\left(z^{0}\right)\right\} .
\end{gathered}
$$

Denote $L^{*}=\max \left\{L\left(z^{0}+t \mathbf{b}\right):|t| \leq \beta / L\left(z^{0}\right)\right\}, \quad \rho_{0}=R / L\left(z^{0}\right), \rho_{k}=\rho_{0}+$ $k \eta / L^{*}, k \in \mathbb{Z}_{+}$. We obtain $\frac{\eta}{L^{*}}<\frac{R}{L^{*}} \leq \frac{R}{L\left(z^{0}\right)}=\frac{\beta}{L\left(z^{0}\right)}-\frac{\beta-R}{L\left(z^{0}\right)}$. Therefore, there exists $n^{*} \in \mathbb{N}$, independent of $z^{0}$ and such that $\rho_{p-1}<\frac{\beta-R}{L\left(z^{0}\right)} \leq \rho_{p} \leq \frac{\beta}{L\left(z^{0}\right)}$, for some $p=p\left(z^{0}\right) \leq n^{*}$. It is possible because $L \in Q_{\mathbf{b}}\left(\mathbb{B}^{n}\right)$. Ar first, one has

$$
\begin{gathered}
\left(\frac{\beta}{L\left(z^{0}\right)}-\rho_{0}\right) /\left(\frac{\eta}{L^{*}}\right)=\frac{(\beta-R) L^{*}}{\eta L\left(z^{0}\right)} \\
=\frac{\beta-R}{\eta} \max \left\{\frac{L\left(z^{0}+t \mathbf{b}\right)}{L\left(z^{0}\right)}:|t| \leq \frac{\beta}{L\left(z^{0}\right)}\right\} \leq \frac{\beta-R}{\eta} \lambda_{\mathbf{b}}(\beta) .
\end{gathered}
$$

Therefore, $n^{*}=\left[\frac{\beta-R}{\eta} \lambda_{\mathbf{b}}(\beta)\right]$, where $[a]$ is an entire part of number $a \in \mathbb{R}$. Let $\left|F\left(z^{0}+t_{k}^{* *} \mathbf{b}\right)\right|=\max \left\{\left|F\left(z^{0}+t \mathbf{b}\right)\right|: t \in c_{k}\right\}, c_{k}=\left\{t \in \mathbb{C}:|t|=\rho_{k}\right\}$, and $t_{k}^{*}$ be the intersection point of the segment $\left[0, t_{k}^{* *}\right]$ with the circle $c_{k-1}$. Hence, for every $r>\eta$ and for each $k \leq n^{*}$ we get the inequality $\left|t_{k}^{* *}-t_{k}^{*}\right|=\frac{\eta}{L^{*}} \leq \frac{r}{L\left(z^{0}+t_{k}^{*} \mathbf{b}\right)}$. Thus, for some $r=r\left(z^{0}+t_{k}^{*} \mathbf{b}\right) \in[\eta, R]$ we deduce

$$
\begin{gathered}
\left|F\left(z^{0}+t_{k}^{* *} \mathbf{b}\right)\right| \leq \max \left\{\left|F\left(z^{0}+t \mathbf{b}\right)\right|:\left|t-t_{k}^{*}\right|=r / L\left(z^{0}+t_{k}^{*} \mathbf{b}\right)\right\} \\
\leq P_{2} \min \left\{\left|F\left(z^{0}+t \mathbf{b}\right)\right|:\left|t-t_{k}^{*}\right|=r / L\left(z^{0}+t_{k}^{*} \mathbf{b}\right)\right\} \\
\leq P_{2} \min \left\{\left|F\left(z^{0}+t \mathbf{b}\right)\right|:\left|t-t_{k}^{*}\right|=r / L\left(z^{0}+t_{k}^{*} \mathbf{b}\right),\left|t-t_{0}\right| \leq \rho_{k-1}\right\} \\
c \leq P_{2} \max \left\{\left|F\left(z^{0}+t \mathbf{b}\right)\right|: t \in c_{k-1}\right\} .
\end{gathered}
$$

Hence,

$$
\begin{gathered}
\max \left\{\left|F\left(z^{0}+t \mathbf{b}\right)\right|:|t|=(\beta-R) / L\left(z^{0}\right)\right\} \\
\leq \max \left\{\left|F\left(z^{0}+t \mathbf{b}\right)\right|: t \in c_{p}\right\} \leq P_{2} \max \left\{\left|F\left(z^{0}+t \mathbf{b}\right)\right|: t \in c_{p-1}\right\} \\
\leq \ldots \leq\left(P_{2}\right)^{p} \max \left\{\left|F\left(z^{0}+t \mathbf{b}\right)\right|: t \in c_{0}\right\} \\
\leq\left(P_{2}\right)^{n^{*}} \max \left\{\left|F\left(z^{0}+t \mathbf{b}\right)\right|:|t|=R / L\left(z^{0}\right)\right\} .
\end{gathered}
$$

We get (16) with $P_{1}=\left(P_{2}\right)^{n^{*}}$. Thus, for $R \in(0, \beta / 2)$ Theorem 8 is proved. 
Now, suppose that $R \in[\beta / 2, \beta)$ and $\left(\forall z \in \mathbb{B}^{n}\right): L(z)>\frac{2 \beta|b|}{1-|z|}$. Then inequality (13) can be rewritten as

$$
\begin{gathered}
\max \left\{\left|F\left(z^{0}+\frac{t}{2} \cdot 2 \mathbf{b}\right)\right|:|t / 2|=\frac{r / 2}{L\left(z^{0}\right)}\right\} \\
\leq P_{2} \min \left\{\left|F\left(z^{0}+\frac{t}{2} \cdot 2 \mathbf{b}\right)\right|:|t / 2|=\frac{r / 2}{L\left(z^{0}\right)}\right\} .
\end{gathered}
$$

Denoting $t^{\prime}=t / 2$, one has

$$
\begin{aligned}
& \max \left\{\left|F\left(z^{0}+t^{\prime} \cdot 2 \mathbf{b}\right)\right|:\left|t^{\prime}\right|=\frac{r / 2}{L\left(z^{0}\right)}\right\} \\
\leq & P_{2} \min \left\{\left|F\left(z^{0}+t^{\prime} \cdot 2 \mathbf{b}\right)\right|:\left|t^{\prime}\right|=\frac{r / 2}{L\left(z^{0}\right)}\right\} .
\end{aligned}
$$

Since $r \leq R \in[\beta / 2, \beta)$, we have $r / 2 \leq R \in[\beta / 4, \beta / 2) \subset(0, \beta / 2)$. Therefore, as shown above the function $F$ has bounded $L$-index in the direction $2 \mathbf{b}$, but by Lemma 2 the function is also of bounded $L$-index in the direction $\mathbf{b}$.

\section{Estimate of directional logarithmic derivative}

In this section we deduce an analog of logarithmic criterion for function from the class $\widetilde{\mathcal{H}}_{\mathbf{b}}\left(\mathbb{B}^{n}\right)$. The one-dimensional analog of the criterion is efficient to investigate boundedness of $l$-index of infinite products $[9,19,18]$. As necessary conditions the criterion was obtained by Fricke $[11,10]$ for entire functions of bounded index.

Below we prove the criterion of $L$-index boundedness in direction, which describes behavior of directional logarithmic derivative and distribution of zeros. We need the additional denotations.

Denote

$$
G_{r}(F):=G_{r}^{\mathbf{b}}(F):=\bigcup_{z: F(z)=0}\{z+t \mathbf{b}:|t|<r / L(z)\} .
$$

By $n\left(r, z^{0}, 1 / F\right)=\sum_{\left|a_{k}^{0}\right| \leq r} 1$ we denote a counting function of zeros $a_{k}^{0}$, where $a_{k}^{0}$ are zeros of the function $F\left(z^{0}+t \mathbf{b}\right)$ for a given $z^{0} \in \mathbb{B}^{n}$.

Theorem 9. Let $F \in \widetilde{\mathcal{H}}_{\mathbf{b}}\left(\mathbb{B}^{n}\right), L \in Q_{\mathbf{b}}\left(\mathbb{B}^{n}\right)$. If the function $F$ has bounded $L$-index in the direction $\mathbf{b}$ then 
1) for every $r \in(0, \beta]$ there exists $P=P(r)>0$ that for each $z \in \mathbb{B}^{n} \backslash G_{r}^{\mathbf{b}}(F)$

$$
\left|\frac{\partial_{\mathbf{b}} F(z)}{F(z)}\right| \leq P L(z)
$$

2) for every $r \in(0, \beta]$ there exists $\widetilde{n}(r) \in \mathbb{Z}_{+}$such that for each $z^{0} \in \mathbb{B}^{n}$ with $F\left(z^{0}+t \mathbf{b}\right) \not \equiv 0$

$$
n\left(r / L\left(z^{0}\right), z^{0}, 1 / F\right) \leq \widetilde{n}(r) .
$$

Proof. Our proof is based on the proof of appropriate proposition for analytic functions of bounded $L$-index in direction [7].

First, we prove that if the function $F(z)$ is of bounded $L$-index in the direction $\mathbf{b}$, then for every $z^{0} \in \mathbb{B}^{n} \backslash G_{r}^{\mathbf{b}}(F)(r \in(0, \beta])$ and for every $\widetilde{a}^{k}=$ $z^{0}+a_{k}^{0} \mathbf{b}$ the following inequality holds

$$
\left|z^{0}-\widetilde{a}_{k}\right|>\frac{r|\mathbf{b}|}{2 L\left(\widetilde{z}^{0}\right) \lambda_{2}^{\mathbf{b}}\left(z^{0}, r\right)} .
$$

On the contrary, we assume that there exist $z^{0} \in \mathbb{B}^{n} \backslash G_{r}^{\mathbf{b}}(F)$ and $\widetilde{a}^{k}=z^{0}+a_{k}^{0} \mathbf{b}$ such that $\left|z^{0}-\widetilde{a}_{k}\right| \leq \frac{r|\mathbf{b}|}{2 L\left(\widetilde{z}^{0}\right) \lambda_{2}^{\mathbf{b}}\left(z^{0}, r\right)} \leq \frac{r|\mathbf{b}|}{2 L\left(z^{0}\right)}<\frac{r|\mathbf{b}|}{L\left(z^{0}\right)}$. Hence, $\left|a_{k}^{0}\right|<\frac{r}{L\left(z^{0}\right)}$. But for $\lambda_{2}^{\mathbf{b}}$ the following estimate holds $L\left(\widetilde{a}^{k}\right) \leq \lambda_{2}^{\mathbf{b}}\left(z^{0}, r\right) L\left(z^{0}\right)$ and $\left|z^{0}-\widetilde{a}^{k}\right|=$ $|\mathbf{b}| \cdot\left|a_{k}^{0}\right| \leq \frac{r|\mathbf{b}|}{2 L\left(\widetilde{a}^{k}\right)}$, i.e. $\left|a_{k}^{0}\right| \leq \frac{r}{2 L\left(\widetilde{a}^{k}\right)}$. It contradicts $z^{0} \in \mathbb{C}^{n} \backslash G_{r}^{\mathbf{b}}(F)$. In fact, in (20) instead of $\lambda_{2}^{\mathbf{b}}\left(z^{0}, r\right)$ we can take $\lambda_{2}^{\mathbf{b}}(r)$.

We choose in Theorem $7 R=\frac{r}{2 \lambda_{2}^{\mathbf{b}}(r)}$. Then there exists $P_{2} \geq 1$ and $\eta \in(0, R)$ such that for every $z^{0} \in \mathbb{B}^{n}$ and some $r^{*} \in[\eta, R]$ inequality (13) holds with $r^{*}$ instead of $r$. Therefore, by Cauchy's inequality

$$
\begin{gathered}
\left|\partial_{\mathbf{b}} F\left(z^{0}\right)\right| \leq \frac{L\left(z^{0}\right)}{r^{*}} \max \left\{\left|F\left(z^{0}+t \mathbf{b}\right):\right| t \mid=\frac{r^{*}}{L\left(z^{0}\right)}\right\} \\
\leq P_{2} \frac{L\left(z^{0}\right)}{\eta} \min \left\{\left|F\left(z^{0}+t \mathbf{b}\right)\right|:|t|=\frac{r^{*}}{L\left(z^{0}\right)}\right\} .
\end{gathered}
$$

In view of (20) the set $\left\{z^{0}+t \mathbf{b}:|t| \leq \frac{r}{2 \lambda_{2}^{\mathbf{b}}(r) L\left(z^{0}\right)}\right\}$ does not contain zeros of the function $F\left(z^{0}+t \mathbf{b}\right)$ for every $z^{0} \in \mathbb{B}^{n} \backslash G_{r}^{\mathbf{b}}(F)$. Therefore, applying the maximum modulus principle to $1 / F$, as a function of $t$, we have

$$
\left|F\left(z^{0}\right)\right| \geq \min \left\{\left|F\left(z^{0}+t \mathbf{b}\right)\right|:|t|=r^{*} / L\left(z^{0}\right)\right\} .
$$


Inequalities (21) and (22) imply (18) with $P=P_{2} / \eta$.

Now we prove that if $F$ is of bounded $L$-index in the direction $\mathbf{b}$ then there exists $P_{3}>0$ such that for every $z^{0} \in \mathbb{B}^{n}\left(F\left(z^{0}+t \mathbf{b}\right) \not \equiv 0\right), r \in(0,1]$

$$
\begin{gathered}
n\left(r / L\left(z^{0}\right), z^{0}, 1 / F\right) \min \left\{\left|F\left(z^{0}+t \mathbf{b}\right)\right|:|t|=r / L\left(z^{0}\right)\right\} \\
\leq P_{3} \max \left\{\left|F\left(z^{0}+t \mathbf{b}\right)\right|:|t|=1 / L\left(z^{0}\right)\right\} .
\end{gathered}
$$

By Cauchy's inequality and Theorem 4 for all $t \in S_{z^{0}}$ such that $|t|=1 / L\left(z^{0}\right)$ we have

$$
\begin{gathered}
\left|\partial_{\mathbf{b}} F\left(z^{0}+t \mathbf{b}\right)\right| \leq \frac{L\left(z^{0}\right)}{\beta-1} \max \left\{\left|F\left(z^{0}\right)\right|:|\theta-t|=\frac{\beta-1}{L\left(z^{0}\right)}\right\} \\
\leq \frac{L\left(z^{0}\right)}{\beta-1} \max \left\{\left|F\left(z^{0}+t \mathbf{b}\right)\right|:|t|=\frac{\beta}{L\left(z^{0}\right)}\right\} \\
\leq \frac{P_{1}(1, \beta)}{\beta-1} L\left(z^{0}\right) \max \left\{\left|F\left(z^{0}+t \mathbf{b}\right)\right|:|t|=\frac{1}{L\left(z^{0}\right)}\right\} .
\end{gathered}
$$

If $F\left(z^{0}+t \mathbf{b}\right) \neq 0$ on a circle $\left\{t \in S_{z^{0}}:|t|=r / L\left(z^{0}\right)\right\}$ then

$$
\begin{aligned}
& n\left(\frac{r}{L\left(z^{0}\right)}, z^{0}, \frac{1}{F}\right)=\left|\frac{1}{2 \pi i} \int_{|t|=\frac{r}{L\left(z^{0}\right)}} \frac{\partial_{\mathbf{b}} F\left(z^{0}+t \mathbf{b}\right)}{F\left(z^{0}+t \mathbf{b}\right)} d t\right| \\
& \leq \frac{\max \left\{\left|\partial_{\mathbf{b}} F\left(z^{0}+t \mathbf{b}\right)\right|:|t|=r / L\left(z^{0}\right)\right\}}{\min \left\{\left|F\left(z^{0}+t \mathbf{b}\right)\right|:|t|=r / L\left(z^{0}\right)\right\}} \frac{r}{L\left(z^{0}\right)} .
\end{aligned}
$$

From (24) and (25) we have

$$
\begin{aligned}
& n\left(r / L\left(z^{0}\right), z^{0}, 1 / F\right) \min \left\{\left|F\left(z^{0}+t \mathbf{b}\right)\right|:|t|=r / L\left(z^{0}\right)\right\} \\
& \leq \frac{r}{L\left(z^{0}\right)} \max \left\{\left|\partial_{\mathbf{b}} F\left(z^{0}+t \mathbf{b}\right)\right|:|t|=r / L\left(z^{0}\right)\right\} \\
& \quad \leq \frac{1}{L\left(z^{0}\right)} \max \left\{\left|\partial_{\mathbf{b}} F\left(z^{0}+t \mathbf{b}\right)\right|:|t|=1 / L\left(z^{0}\right)\right\} \\
& \leq P_{1}(1, \beta) /(\beta-1) \max \left\{\left|F\left(z^{0}+t \mathbf{b}\right)\right|:|t|=1 / L\left(z^{0}\right)\right\} .
\end{aligned}
$$

Thus, we obtain $(23)$ with $P_{3}=P_{1}(1, \beta) /(\beta-1)$. If the function $F\left(z^{0}+t \mathbf{b}\right)$ has zeros on the circle $\left\{t \in D_{R}^{z^{0}}:|t|=r / L\left(z^{0}\right)\right\}$, then inequality (23) is obvious.

Now we put $R=1$ in Theorem 7 . Then there exists $P_{2}=P_{2}(1) \geq 1$ and $\eta \in(0,1)$ such that for each $z^{0} \in \mathbb{B}^{n}$ and some $r^{*}=r^{*}\left(z^{0}\right) \in[\eta, 1]$

$$
\max \left\{\left|F\left(z^{0}+t \mathbf{b}\right)\right|:|t|=\frac{r^{*}}{L\left(z^{0}\right)}\right\} \leq P_{2} \min \left\{\left|F\left(z^{0}+t \mathbf{b}\right)\right|:|t|=\frac{r^{*}}{L\left(z^{0}\right)}\right\} .
$$


Moreover, by Theorem 4 there exists $P_{1} \geq 1$ such that for all $z^{0} \in \mathbb{B}^{n}$

$$
\begin{gathered}
\max \left\{\left|F\left(z^{0}+t \mathbf{b}\right)\right|:|t|=1 / L\left(z^{0}\right)\right\} \\
\leq P_{1}(1, \eta) \max \left\{\left|F\left(z^{0}+t \mathbf{b}\right)\right|:|t|=\eta / L\left(z^{0}\right)\right\} \\
\leq P_{1}(1, \eta) \max \left\{\left|F\left(z^{0}+t \mathbf{b}\right)\right|:|t|=r^{*} / L\left(z^{0}\right)\right\} \\
\leq P_{1}(1, \eta) P_{2} \min \left\{\left|F\left(z^{0}+t \mathbf{b}\right)\right|:|t|=r^{*} / L\left(z^{0}\right)\right\} .
\end{gathered}
$$

Taking into account (23), we have

$$
\begin{gathered}
n\left(\frac{r^{*}}{L\left(z^{0}\right)}, z^{0}, \frac{1}{F}\right) \min \left\{\left|F\left(z^{0}+t \mathbf{b}\right)\right|:|t|=\frac{r^{*}}{L\left(z^{0}\right)}\right\} \\
\leq P_{3} P_{1} P_{2} \min \left\{\left|F\left(z^{0}+t \mathbf{b}\right)\right|:|t|=\frac{r^{*}}{L\left(z^{0}\right)}\right\}
\end{gathered}
$$

i.e. $n\left(\frac{r^{*}}{L\left(z^{0}\right)}, z^{0}, \frac{1}{F}\right) \leq P_{1}(1, \eta) P_{2} P_{3}$. Hence,

$$
n\left(\frac{r^{*}}{L\left(z^{0}\right)}, z^{0}, \frac{1}{F}\right) \leq P_{4}=P_{1}(1, \eta) P_{2} P_{3}=\frac{P_{1}(1, \eta) P_{2}(1) P_{1}(1, r+1)}{r} .
$$

If $r \in(0, \eta]$ then property (19) is proved.

Let $r \in(\eta, \beta]$ and $L^{*}=\max \left\{L\left(z^{0}+t \mathbf{b}\right):|t|=\frac{r}{L\left(z^{0}\right)}\right\}$. Using properties of $Q_{\mathbf{b}}^{n}$, we have $L^{*} \leq \lambda_{2}^{\mathbf{b}}(r) L\left(z^{0}\right)$. Put $\rho=\frac{\eta}{L\left(z^{0}\right) \lambda_{2}^{\mathbf{b}}(r)}, R=\frac{r}{L\left(z^{0}\right)}$. We can cover every set $\bar{K}=\left\{z^{0}+t \mathbf{b}:|t| \leq R\right\}$ by a finite number $m=m(r)$ of closed sets $\bar{K}_{j}=\left\{z^{0}+t \mathbf{b}:\left|t-t_{j}\right| \leq \rho\right\}$, where $t_{j} \in \bar{K}$. Since $\frac{\eta}{\lambda_{2}^{\mathbf{b}}(r) L\left(z^{0}\right)} \leq \frac{\eta}{L^{*}} \leq$ $\frac{\eta}{L\left(z^{0}+t_{j} \mathbf{b}\right)}$ in each $\bar{K}_{j}$ there are at most $\left[P_{4}\right]$ zeros of function $F\left(z^{0}+t \mathbf{b}\right)$. Thus, $n\left(\frac{r}{L\left(z^{0}\right)}, z^{0}, 1 / F\right) \leq \widetilde{n}(r)=\left[P_{4}\right] m(r)$ and property $(19)$ is proved.

By $n_{z^{0}}(r, F)=n_{\mathbf{b}}\left(r, z^{0}, 1 / F\right):=\sum_{\left|a_{k}^{0}\right| \leq r} 1$ we denote counting function of zeros $a_{k}^{0}$ for the slice function $F\left(z^{0}+t \mathbf{b}\right)$ in the disc $\{t \in \mathbb{C}:|t| \leq r\}$. If for a given $z^{0} \in \mathbb{C}^{n}$ and for all $t \in \mathbb{C} F\left(z^{0}+t \mathbf{b}\right) \equiv 0$, then we put $n_{z^{0}}(r)=-1$. Denote $n(r)=\sup _{z \in \mathbb{C}^{n}} n_{z}(r / L(z))$.

Theorem 10. Let $L \in Q_{\mathbf{b}}\left(\mathbb{B}^{n}\right), F \in \widetilde{\mathcal{H}}_{\mathbf{b}}\left(\mathbb{B}^{n}\right), \mathbb{B}^{n} \backslash G_{\beta}^{\mathbf{b}}(F) \neq \emptyset$. If the following conditions are satisfied:

1) there exists $r_{1} \in(0, \beta / 2)$ (either there exists $r_{1} \in[\beta / 2, \beta)$ and $\left(\forall z \in \mathbb{B}^{n}\right)$ : $\left.L(z)>\frac{2 \beta|b|}{1-|z|}\right)$ such that $n\left(r_{1}\right) \in[-1 ; \infty)$; 
2) there exist $r_{2} \in(0, \beta), P>0$ such that $2 r_{2} \cdot n\left(r_{1}\right)<r_{1} / \lambda_{\mathbf{b}}\left(r_{1}\right)$ and for all $z \in \mathbb{B}^{n} \backslash G_{r_{2}}(F)$ inequality (18) is true;

then the function $F$ has bounded $L$-index in the direction $\mathbf{b}$.

Proof. Our proof is based on the proof of appropriate proposition for analytic functions of bounded $L$-index in direction [5]. Suppose that conditions 1) and 2) are true.

At first, we consider the case $n\left(r_{1}\right) \in\{-1 ; 0\}$. Then in the best case the function $F$ can only identically equals zero on the complex line $z^{*}+t \mathbf{b}$ for some $z^{*} \in \mathbb{B}^{n}$, i.e., $F\left(z^{*}+t \mathbf{b}\right) \equiv 0$. For all points lying on such complex lines inequality (13) is obvious.

Let $z^{0} \in \mathbb{B}^{n} \backslash G_{r_{2}}$. For any points $t_{1}$ and $t_{2}$ such that $\left|t_{j}\right|=\frac{r_{2}}{L\left(z_{0}\right)}, j \in\{1,2\}$, one has

$$
\begin{gathered}
\ln \left|\frac{F\left(z^{0}+t_{2} \mathbf{b}\right)}{F\left(z^{0}+t_{1} \mathbf{b}\right)}\right| \leq \int_{t_{1}}^{t_{2}}\left|\frac{\partial_{\mathbf{b}} F\left(z^{0}+t \mathbf{b}\right)}{F\left(z^{0}+t \mathbf{b}\right)}\right||d t| \\
\leq P \int_{t_{1}}^{t_{2}} L\left(z^{0}+t \mathbf{b}\right)|d t| \leq P \lambda_{\mathbf{b}}\left(r_{2}\right) L\left(z^{0}\right) \frac{\pi r_{2}}{L\left(z^{0}\right)} \leq \pi r_{2} P \lambda_{\mathbf{b}}\left(r_{2}\right)
\end{gathered}
$$

(we also use that $L \in Q_{\mathbf{b}}\left(\mathbb{B}^{n}\right)$ ). Hence,

$$
\begin{gathered}
\max \left\{\left|F\left(z^{0}+t \mathbf{b}\right)\right|:|t|=\frac{r_{2}}{L\left(z^{0}\right)}\right\} \\
\leq P_{2} \min \left\{\left|F\left(z^{0}+t \mathbf{b}\right)\right|:|t|=\frac{r_{1}}{L\left(z^{0}\right)}\right\},
\end{gathered}
$$

where $P_{2}=\exp \left\{\pi r_{2} P \lambda_{2}\left(r_{2}\right)\right\}$. Therefore, by Theorem 8 the function $F$ has bounded $L$-index in the direction $\mathbf{b}$.

Let $r_{1}>0$ be a such that $n\left(r_{1}\right) \in[1 ; \infty)$ and $2 n\left(r_{1}\right) r_{2}<r_{1} / \lambda_{\mathbf{b}}\left(r_{1}\right)$. Put $c=\frac{r_{1}}{2 r_{2} \lambda_{\mathbf{b}}\left(r_{1}\right)}-n\left(r_{1}\right)>0$. Clearly, $r_{2}=\frac{r_{1}}{2\left(n\left(r_{1}\right)+c\right) \lambda_{\mathbf{b}}\left(r_{1}\right)}$.

Under condition 1) each set $\bar{K}=\left\{z^{0}+t \mathbf{b}:|t| \leq \frac{r_{1}}{L\left(z^{0}\right)}\right\}$ has no more $n\left(r_{1}\right)$ zeros of the function $F$, where $F\left(z^{0}+t \mathbf{b}\right) \not \equiv 0$.

Under condition 2) there exists $P>0$ such that $\left|\frac{\partial_{\mathbf{b}} F(z)}{F(z)}\right| \leq P L(z)$ for every $z \in \mathbb{B}^{n} \backslash G_{r_{2}}$, i.e., for all $z \in \bar{K}$, lying outside the sets $\left\{z^{0}+t \mathbf{b}:\left|t-a_{k}^{0}\right|<\frac{r_{2}}{L\left(z^{0}+a_{k}^{0} \mathbf{b}\right)}\right\}$, where $a_{k}^{0} \in \bar{K}$ are zeros of the slice function $F\left(z^{0}+t \mathbf{b}\right) \not \equiv 0$. By definition $\lambda_{\mathbf{b}}$ we obtain $L\left(z^{0}\right) / \lambda_{\mathbf{b}}\left(r_{1}\right) \leq L\left(z^{0}+a_{k}^{0} \mathbf{b}\right)$. Then $\left|\frac{\partial_{\mathbf{b}} F(z)}{F(z)}\right| \leq P L(z)$ for every point $z \in \mathbb{B}^{n}$, lying outside union of the sets

$$
c_{k}^{0}=\left\{z^{0}+t \mathbf{b}:\left|t-a_{k}^{0}\right| \leq \frac{r_{2} \lambda_{\mathbf{b}}\left(r_{1}\right)}{L\left(z^{0}\right)}=\frac{r_{1}}{2\left(n\left(r_{1}\right)+c\right) L\left(z^{0}\right)}\right\} .
$$


The total sum of diameters of the sets $c_{k}^{0}$ does not exceed the value $\frac{r_{1} n\left(r_{1}\right)}{\left(n\left(r_{1}\right)+c\right) L\left(z^{0}\right)}<$ $\frac{r_{1}}{L\left(z^{0}\right)}$. Hence, there exists a set $\widetilde{c}^{0}=\left\{z^{0}+t \mathbf{b}:|t|=\frac{r}{L\left(z^{0}\right)}\right\}$, where $\frac{r_{1} \min \{1, c\}}{2\left(n\left(r_{1}\right)+c\right)}=$ $\eta<r<r_{1}$, such that, for all $z \in \widetilde{c}^{0}$ one has $\left|\frac{\partial_{\mathbf{b}} F(z)}{F(z)}\right| \leq P L(z) \leq P \lambda_{\mathbf{b}}(r) L\left(z^{0}\right) \leq$ $P \lambda_{\mathbf{b}}\left(r_{1}\right)$ For any points $z_{1}=z^{0}+t_{1} \mathbf{b}$ and $z_{2}=z^{0}+t_{2} \mathbf{b}$ with $\widetilde{c}^{0}$ one has

$$
\begin{aligned}
& \ln \left|\frac{F\left(z^{0}+t_{2} \mathbf{b}\right)}{F\left(z^{0}+t_{1} \mathbf{b}\right)}\right| \leq \int_{t_{1}}^{t_{2}}\left|\frac{\partial_{\mathbf{b}} F\left(z^{0}+t \mathbf{b}\right)}{F\left(z^{0}+t \mathbf{b}\right)}\right||d t| \\
& \leq P \lambda_{2}\left(r_{1}\right) L\left(z^{0}\right) \frac{\pi r}{L\left(z^{0}\right)} \leq \pi r_{1} P\left(r_{2}\right) \lambda_{\mathbf{b}}\left(r_{1}\right) .
\end{aligned}
$$

Therefore,

$$
\max \left\{\left|F\left(z^{0}+t \mathbf{b}\right)\right|:|t|=\frac{r}{L\left(z^{0}\right)}\right\} \leq P_{2} \min \left\{\left|F\left(z^{0}+t \mathbf{b}\right)\right|:|t|=\frac{r}{L\left(z^{0}\right)}\right\}
$$

where $P_{2}=\exp \left\{\pi r_{1} P\left(r_{2}\right) \lambda_{\mathbf{b}}\left(r_{1}\right)\right\}$. If $F\left(z^{0}+t \mathbf{b}\right) \equiv 0$, then inequality (26) is obvious. By Theorem 8 the function $F(z)$ has bounded $L$-index in the direction b. Theorem 10 is proved.

\section{Acknowledgements}

This research was funded by the National Research Foundation of Ukraine, 2020.02/0025, 0120U103996.

\section{References}

[1] A. Bandura, M. Martsinkiv, O. Skaskiv, Slice holomorphic functions in the unit ball having a bounded $L$-index in direction, Axioms, 10, No 1 (2021), Article ID 4; doi: 10.3390/axioms10010004.

[2] A. Bandura, O. Skaskiv, L. Smolovyk, Slice holomorphic solutions of some directional differential equations with bounded $L$-index in the same direction, Demonstr. Math., 52, No 1 (2019), 482-489; doi: 10.1515/dema2019-0043.

[3] A. Bandura, O. Skaskiv, Slice holomorphic functions in several variables with bounded L-index in direction, Axioms, 8, No 3 (2019), Article ID 88; doi: 10.3390/axioms8030088. 
[4] A.I. Bandura, O.B. Skaskiv, Some criteria of boundedness of the $L$-index in direction for slice holomorphic functions of several complex variables, $J$. Math. Sci., 244, No 1 (2020), 1-21; doi: 10.1007/s10958-019-04600-7.

[5] A.I. Bandura, Some weaker sufficient conditions of $L$-index boundedness in direction for functions analytic in the unit ball, Carpathian Math. Publ., 11, No 1 (2019), 14-25; doi:10.15330/cmp.11.1.14-25.

[6] A. Bandura, O. Skaskiv, Sufficient conditions of boundedness of Lindex and analog of Hayman's Theorem for analytic functions in a ball, Stud. Univ. Babeş-Bolyai Math., 63, No 4 (2018), 483-501; doi:10.24193/subbmath.2018.4.06.

[7] A. Bandura, O. Skaskiv, Functions analytic in the unit ball having bounded L-index in a direction, Rocky Mountain J. Math., 49, No 4 (2019), 10631092; doi: 10.1216/RMJ-2019-49-4-1063.

[8] A.I. Bandura, Some improvements of criteria of $L$-index boundedness in direction, Mat. Stud., 47, No 1 (2017), 27-32; doi: 10.15330/ms.47.1.27-32.

[9] M.T. Bordulyak, M.M. Sheremeta, On the existence of entire functions of bounded $l$-index and $l$-regular growth, Ukrainian Math. J., 48, No 9 (1996), 1322-1340; doi: 10.1007/BF02595355.

[10] G.H. Fricke, Functions of bounded index and their logarithmic derivatives, Math. Ann., 206 (1973), 215-223.

[11] G.H. Fricke, Entire functions of locally slow growth, J. Anal. Math., 28, No 1 (1975), 101-122.

[12] A.D. Kuzyk, M.N. Sheremeta, On entire functions, satisfying linear differential equations, Diff. Equations, 26, No 10 (1990), 1716-1722.

[13] F. Nuray, Bounded index and four dimensional summability methods, Novi Sad J. Math., 49 (2019), 73-85; doi:10.30755/NSJOM.08285.

[14] F. Nuray, R.F. Patterson, Vector-valued bivariate entire functions of bounded index satisfying a system of differential equations, Mat. Stud., 49, No 1 (2018), 67-74 (2018); doi: 10.15330/ms.49.1.67-74.

[15] M.N. Sheremeta, A.D. Kuzyk, Logarithmic derivative and zeros of an entire function of bounded l-index, Sib. Math. J., 33, No 2 (1992), 304-312; doi: 10.1007/BF00971102. 
[16] M. Sheremeta, Analytic Functions of Bounded Index, VNTL Publishers, Lviv (1999).

[17] M.N. Sheremeta, An $l$-index and an $l$-distribution of the values of entire functions, Soviet Math. (Iz. VUZ), 34 No 2 (1990), 115-117.

[18] M.M. Sheremeta, Generalization of the Fricke theorem on entire functions of finite index, Ukrainian Math. J., 48, No 3 (1996), 460-466; doi: $10.1007 / \mathrm{BF} 02378535$.

[19] M.M. Sheremeta, M.T. Bordulyak, Boundedness of the $l$-index of LaguerrePolya entire functions, Ukr. Math. J., 55, No 1 (2003), 112-125; doi: 10.1023/A:1025076720052. 
